\section{P2.166 THE EFFECTIVENESS OF SMS TEXTS FOR REMINDING PATIENTS AT HIGH RISK OF STIS AND HIV TO RETURN FOR TESTING}

doi:10.1136/sextrans-2013-051184.0430

G Brook, J Burton, J McSorley, S Murphy. Central Middlesex Hospital, London, UK

Background Patients attending sexual health services for STI/HIV testing may be at continuing risk of infection acquisition or may be within a window period for testing and may be advised to return for retesting at various intervals. Technology such as SMS texting facilitates the sending of reminders.

Objectives To measure the impact of the SMS text reminders on the re-attendance rates of patients who require repeat testing.

Methods Re-attendance rates were measured for patients listed for routine SMS text reminders (September to November 2012) and a control group with the same risk factors from a period when text reminders were not routinely used (September to November 2011). Re-attendance was counted if it was within 2 months of the text or, for the control group, within 3 months of the end of the previous episode of care.

Results Following the introduction of SMS text reminders, the reattendance rate was $41 \%(84 / 207)$ in the text group compared to $28 \%(47 / 169)$ in the control group to $\mathrm{P}<0.001$. The risk groups most likely to respond to texting were those patients in the window period for HIV $(10 / 33,30 \%$ vs $4 / 26.15 \% \mathrm{P}<0.05)$, women who had received emergency contraception $(25 / 54.46 \%$ vs $15 / 43,35 \%$ $\mathrm{P}<0.05)$ and MSM not in other risk categories (8/17, $47 \%$ vs $2 / 16$, $13 \% \mathrm{P}<0.05)$. No differences were observed in the return rate for patients with other risks (recent acute STIs, syphilis, recent viral hepatitis and sex workers).

Conclusions SMS texts sent as reminders to patients at higher risk of STIs and HIV increases the overall re-attendance rate by $13 \%$ and was especially effective for MSM, people in the window period for $\mathrm{HIV}$ and women who had received emergency contraception. Routine text reminders are an effective, cheap and simple way of ensuring that high-risk patients return for testing.

\section{P2.167 COMPUTER SELF-INTERVIEW INCREASES THE IDENTIFICATION OF PREVIOUSLY HIV-DIAGNOSED STD CLINIC PATIENTS WHO ARE OUT OF HIV CARE}

doi:10.1136/sextrans-2013-051184.0431

1,2J C Dombrowski, 1,2M R Golden. 'University of Washington, Seattle, WA, United States; ${ }^{2}$ Public Health - Seattle \& King County, Seattle, WA, United States

Background STD Clinics are one venue in which to identify HIVdiagnosed persons disengaged from HIV care.

Methods We analysed data from previously HIV-diagnosed patients seen in the STD Clinic in Seattle, Washington, USA, October 2010-December 2012. The clinic uses a computer-assisted selfinterview (CASI) during new problem visits, which queries HIV-diagnosed patients about their HIV care history. Clinicians interview patients who do not complete the CASI. We used chisquared tests to compare the reasons for STD Clinic visits and HIV transmission risk factors in visits at which patients were nonengaged (self-reported out of care or $>6$ month gap in care) compared to engaged in HIV care.

Results Of 1369 visits among previously diagnosed HIV-infected persons, 913 (67\%) included CASI. CASI visit patients were younger (median age 40 vs. 44 years, $\mathrm{p}<0.001)$ and more often MSM $(96 \%$ vs. $88 \%, p<0.001)$ than non-CASI visit patients. Of the $1233(90 \%)$ visits with HIV care data, $20 \%$ were non-engaged. More CASI than non-CASI visits were ascertained as non-engaged (22\% vs. $11 \%$; $p<0.001)$. Symptoms were the visit reason more frequently at non-engaged than engaged visits ( $47 \%$ vs. $40 \%$; $p=0.001$ ), and nonengaged visits were more likely to result in a diagnosis of gonorrhoea ( $27 \%$ vs. $17 \%, p<0.001)$ or syphilis $(13 \%$ vs. $9 \%, p=0.04)$. Although patients reported unprotected anal intercourse with HIV-negative or unknown status partners (nonconcordant UAI) in the past year in $36 \%$ of both non-engaged and engaged visits, self-report of a detectable serum HIV RNA level plus nonconcordant UAI was more frequent in non-engaged than engaged visits (23\% vs. $11 \%$; $\mathrm{p}<0.001$ ).

Conclusions Many persons who are disengaged from HIV care seek evaluation in STD clinics. These patients are at elevated risk for transmitting HIV. Computer-based efforts to assess HIV care engagement increase identification of out-of-care persons, an important first step for re-linkage to HIV care.

\section{P2.168 MANAGEMENT OF SEXUALLY TRANSMITTED INFECTIONS BY AUSTRALIAN GENERAL PRACTITIONERS}

doi:10.1136/sextrans-2013-051184.0432

A Santella, A Pollack, C Harrison, S Sawleshwarkar, H Britt, R Hillman. University of Sydney, Parramatta, Australia

Background In Australia, General Practitioners (GPs) are the gatekeepers to the health care system and about $85 \%$ of the population attends a GP in any one year. This study aimed to determine how frequently six common Sexually Transmitted Infections (STIs) were managed by GPs and the characteristics of the GPs and patients at these encounters.

Methods Data from the Bettering the Evaluation and Care of Health $(\mathrm{BEACH})$ programme were analysed. BEACH is a continuous, paper-based, national study of GP activity in Australia. GPpatient encounters during which at least one STI was managed were identified. The management rates per 100,000 encounters were calculated for April 2000 to March 2012.

Results Between April 2000 and March 2012, 11,784 GPs took part in the BEACH study, collectively recording $1,178,400$ encounters with patients. There were 3,835 STIs encounters recorded, managed at an overall rate of 325 STIs per 100,000 encounters, (compared, for example, to 1,691 non-venereal lower urinary tract infections per 100,000 encounters), comprising 115 herpes problems per 100,000 encounters, 92 genital warts, $67 \mathrm{HIV}, 39$ chlamydia, 6 gonorrhoea and 7 syphilis. Higher management rates occurred among patients who were: male; $15-24$ years old; more socially advantaged; Aboriginal/Torres Strait Islander; resident in a major city; and/or of English-speaking background. GPs who were female or aged less than 60 years had higher STI management rates than their counterparts.

Conclusion This study estimates the management frequency of six common STIs in Australian general practise and the associated patient and GP characteristics. Moving forward, sexual health care in Australia is expected to rely heavily on the continued participation of general practise, with sexual health clinics focusing on priority, high-risk populations. This study provides a basis for priorities in GP workforce development, populationbased health promotion, access, and health services planning strategies.

\section{P2.169 USING BIOMETRIC TECHNOLOGY TO TRACK TB-HIV CO-INFECTED PATIENTS}

doi:10.1136/sextrans-2013-051184.0433

S Batra, S Ahuja, A Sinha, N Gordon. Operation ASHA, Sarita Vihar, New Delhi, India

Background It is estimated that there are over one million people co-infected with tuberculosis (TB) and HIV and multi-drug resistant tuberculosis (MDR-TB) has been shown to be twice as 
common in HIV patients. Because TB and HIV have long treatment durations and are both vulnerable to drug resistance, Operation ASHA's adherence model has demonstrated that it is capable of fighting drug resistant TB, HIV/AIDS, and TB-HIV co-infections.

Methods To fight the rise of MDR-TB, Operation ASHA developed eCompliance with Microsoft Research and Innovators in Health. eCompliance is a biometric terminal that allows health programmes to track patient adherence to medicines over long periods of time. The system updates a central online database on a daily basis through SMS. This combination of biometric and mobile technology has digitised attendance logs and has reduced the response time for counsellors to address defaulting patients.

Results The system has been proven to dramatically improve patient adherence to tuberculosis treatment, and the concept is being utilised to directly address the problem MDR-TB and HIV.

Conclusion This presentation discusses the effectiveness of eCompliance to dispense second-line MDR-TB medicines and ARVs.

\section{P2.170 OUTCOMES OF ISONIAZID PROPHYLAXIS AMONG HIV- INFECTED CHILDREN IN ROUTINE CLINICAL SETTINGS IN KENYA}

doi:10.1136/sextrans-2013-051184.0434 and WHO report 2011).If no effort done, at least 67 children will be exposed to HIV every day. As a measure of prevention TASO adopted the strategy of early infant diagnosis.

Description The introduction of a PMTCT clinic day with trained staffs, to offer a specialised care to pregnant women, post natal mothers and exposed infants. 299 exposed infants were done DNAPCR at 6weeks of age. This was done between November 2010 to May 2012. Mothers were counselled on infant feeding options, those on ART continued, both pregnant women and infants received ARV prophylaxis, follow up visit were done. This was all done in the PMTCT clinic. Both A and B options of PMTCT were used.

Results Out of the 299 infants who were done DNA-PCR, $283(93 \%)$ were DNA-PCR negative, 18 (6\%) were DNA-PCR positive. Those infected were started on HAART. Lesson learned Easy monitoring, virtual elimination is possible, minimal lost to follow up, easy follow up, collective effort is needed, and many exposed infants are saved from the infection.

Conclusion The way to go is set a PMTCT clinic day, and do early infant diagnosis.

\section{P2.172 AUDIT OF MANAGING SAFEGUARDING RISKS IN YOUNG PEOPLE PRESENTING TO OUR CONTRACEPTION AND SEXUAL HEALTH SERVICES}

doi:10.1136/sextrans-2013-051184.0436

E Masini, W Herman, S Joseph. Ministry of Health, Division of TB, NAIROBI, Kenya

Background In 2011, the TB and HIV control programmes commenced a phased isoniazid prophylaxis therapy (IPT) implementation in selected public hospitals in Kenya. We report outcomes from the first three public health facilities' HIV care clinics to provide this service. We established rates of treatment completion, loss-to-follow up, mortality, adverse drug reactions and $\mathrm{TB}$ disease among HIV-infected children during 6 months of IPT.

Methods A retrospective records review of all HIV-infected children (1-14 years) enrolled on IPT from 1st september 2011 and had completed by 30 th november 2012. They were screened for TB using a standardised symptom tool developed by the Division of Leprosy, TB and Lung Disease, Kenya.

Results We reviewed 606 children, 93.7\% were on HAART. IPT successfully completed by $556(92 \%)$ children. Four $(0.7 \%)$ were lost-to-follow up and treatment interruptions of 5-33 days reported in 24 children (4\%). Twenty four children (4\%) had treatment interruption of 5-33 days; twenty of them were assigned an equivalent number of days to cover for the interruption while the rest (4) were among those discontinued. Adverse drug reactions and deaths were reported in 2 children $(0.3 \%)$ respectively. Prophylaxis was discontinued in 24 (4\%) children for various reasons. This includes 18 children $(3 \%)$ diagnosed with TB in a median time of 3 weeks post IPT initiation. $77.8 \%$ of all the children who developed TB while on IPT had advanced HIV disease at baseline. Isoniazid resistance was not detected in the four culture confirmed tuberculosis cases.

Conclusion The findings of high treatment completion, low lossto-follow up rate and few adverse drug reactions affirm the feasibility and safety of IPT provision to HIV-infected children in routine settings, but incident TB during the first weeks suggests inadequacy of the symptom screening tool in severely immune-compromised children.

\section{P2.171 VIRTUAL ELIMINATION OF PAEDIATRIC HIV}

doi:10.1136/sextrans-2013-051184.0435

C M Ngelese. The AIDS support organization Uganda, Kampala, Uganda

Background HIV disease continues to advance in way that it infects nine people every other minute. It is also estimated that it will claim 3.1 million this year, and 570,000 are children (UN aid,
L Anobah, R E Browne. St Anns Sexual Health Centre, London, UK

Background There is concern about the increasing risk of young people to sexual exploitation and it is essential that services which young people may self refer to have systems in place to ensure that if vulnerable young people attend these risks are detected and acted on. Aim We aimed to evaluate whether young people accessing our services are being adequately assessed for safeguarding risks and whether these are being addressed appropriately within our services. Method We identified all patients aged $<16$ years who attended our clinics over a 6 month period (February - August 2012). Date was collected from electronic patient records (EPR) and analysed using an Excel spreadsheet.

There were 336 attendees of which 78 were new patients and these were audited. The median age was 15 years (range 13-15 years). 77 were heterosexual. There was clear full documentation in 65 patients. The main reason for attendance were contraception, STI screening, pregnancy tests and termination of pregnancy referrals. 79\% (62) were sexually active and the median number of partners in the preceding 3 months was 1 (range $0-7$ ). The median partner's age was 15 (range 14-22) years. The median age differential was 1 year (range 3-8 years). 74\% (58) had documented Fraser competency assessment and this increased to $100 \%(56 / 56)$ where a young person proforma was used. 57 were Fraser competent. 13 cases had safeguarding concerns and their management will be discussed.

Recommendations Were to ensure all $<16$ year olds are managed using the clinic's young person's proforma, all have a documented assessment of Fraser competency and safeguarding risks, patients with risks are highlighted in EPR and monthly supervision is done

P2.173 PREGNANCY AND CONTRACEPTION: THE PERSPECTIVE OF HIV-POSITIVE AND NEGATIVE WOMEN

doi:10.1136/sextrans-2013-051184.0437

C 0 Oraka. Action Group on Adolescent Health, Nnamdi Azikiwe University Teaching Hospital, Nnewi, Nigeria

Objective To understand pregnancy intentions and contraception knowledge and use among HIV-positive and negative women in the
54 were in school and 13 reported involvement of social services. to share best practise. 